\title{
Selection of optimum structural systems and materials
}

\author{
O. S. Al Shamrani \& G. G. Schierle \\ USC School of Architecture, Los Angeles, USA
}

\begin{abstract}
This paper proposes procedures and guidelines for the selection of optimum structural systems and materials in two stages. Stage one is based on a list of criteria, including architectural considerations. Stage two evaluates selected systems and materials for optimum performance of criteria considered critical for a given project. A tall office building in Dammam, Saudi Arabia is used as a case study to compare three structural systems: moment frame, braced frame, and shear wall; as well as two materials: concrete and steel. The case study considers four building heights: 10, 20, 30, and 40 stories. The STAAD Pro 2005 software is used to analyze these systems according to allowable stress requirements for an objective function to minimize drift, at minimal cost for a wind speed of $90 \mathrm{mph}$. Shear wall is the optimum structural system and concrete the optimum material to minimize lateral drift at minimum material and labor costs.
\end{abstract}

Keywords: lateral drift, wind load, IBC 03, allowable stress, STAAD Pro.

\section{Introduction}

The selection of structural system and material is often done according to personal experience or perception without being evaluated as it should be for optimum performance. The proposed selection process provides a methodology to determine the selection of the optimum structural system. Since in Saudi Arabia wind load is more critical than seismic load, this paper investigates wind effect on tall buildings. Since wind load increases with height on tall buildings and causes lateral deflection (drift), minimizing lateral drift is an important criterion to select structural systems for tall buildings. Controlling drift is vital 
to provide occupant comfort and avoid motion sickness. Furthermore, large drift may endanger life and incur loss of property or even cause building collapse.

\section{Methodology}

Stage one includes, among others, consideration of the following criteria: gravity load, lateral load (wind and seismic), climate conditions, labor and material costs, code requirements, building location, building height limit, sustainability (durable and recyclable), strength, stiffness, stability, and synergy. For example, building location is a significant criterion which affects the selection of material. In Saudi Arabia, the most popular building material is concrete; while wood is not available and steel is very expensive.

Stage two is defined by the process shown in Figure 1. For example, considering two materials (steel and concrete), three structural systems (moment frame, braced frame, and shear wall); this process implies four combinations of structural systems and materials for each building height. Each material and system combination is passed through a criteria process to minimize lateral drift (main criterion) as well as minimizing labor and material costs (secondary criterion). All material and structural system combinations are entered to the design evaluation of STAAD Pro 2005. Comparing all results leads to the selection of the optimum structural system and material.

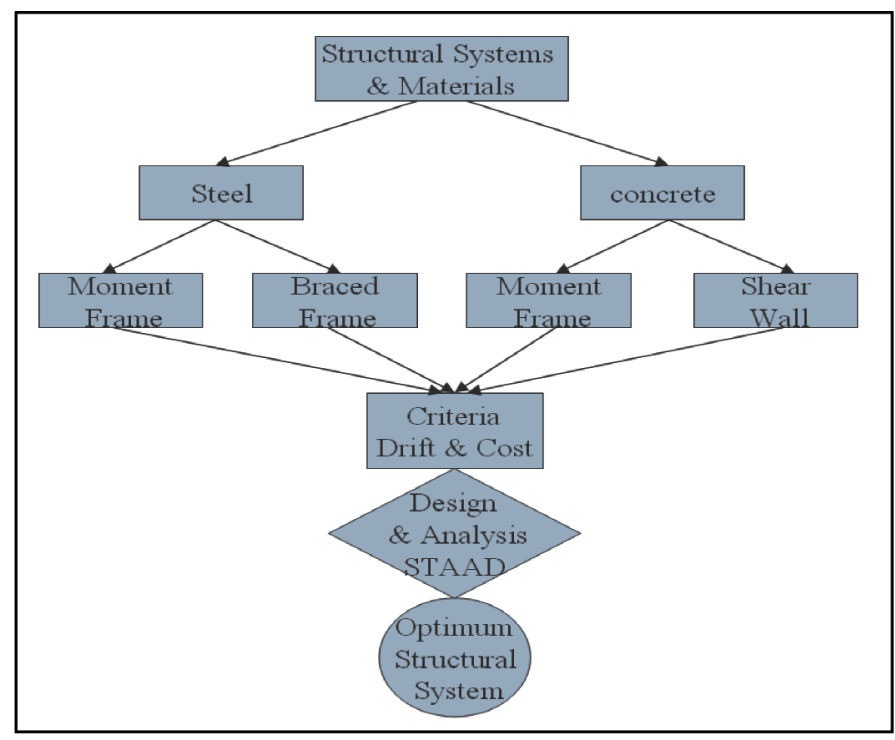

Figure 1: $\quad$ Selection of the optimum structural system process. 


\section{Assumptions}

Using the structural design and analysis software (STAAD Pro), the case study assumed: International Building Code IBC 03; $90 \mathrm{mph}$ maximum wind speed; allowable stress design method for schematic structural design; material strength: concrete $9.0 \mathrm{ksi}$, steel: $50 \mathrm{ksi}$. Structural members are designed to meet the allowable stresses, using safety factors of $45 \%$ for concrete and $60 \%$ for steel (4 ksi concrete, $30 \mathrm{ksi}$ steel beam; $25 \mathrm{ksi}$ steel columns due to buckling) and maximum allowable lateral drift (h/200). Gravity load was applied simultaneously with wind load, assuming combined dead and live loads uniform distributed loads of $1.7 \mathrm{kip} / \mathrm{ft}$. Lateral wind load per level (in kips) was assumed acting on wind and lee sides as shown in Figs. 2-5.

Variables investigated in the case study:

- $\quad$ Building height (10, 20, 30, and 40 stories)

- Structural systems (moment frame, braced frame, and shear wall)

- $\quad$ Structural materials (concrete and steel)

Those variables combined create 16 cases.

The lateral drift is measured at each level after defining member size for strength to assure the actual lateral drift is less than the maximum allowable. Labor and material cost for each system is based on current costs in Saudi Arabia. The tested case study consists of 3 bay frames of $33 \times 33 \mathrm{ft}(10 \times 10 \mathrm{~m})$ column spacing and $13 \mathrm{ft}(4 \mathrm{~m})$ story height. Member sizes are based on combined wind and gravity loads. Beam and column sizes vary every two floors as shown in the tables.

\section{10-story building}
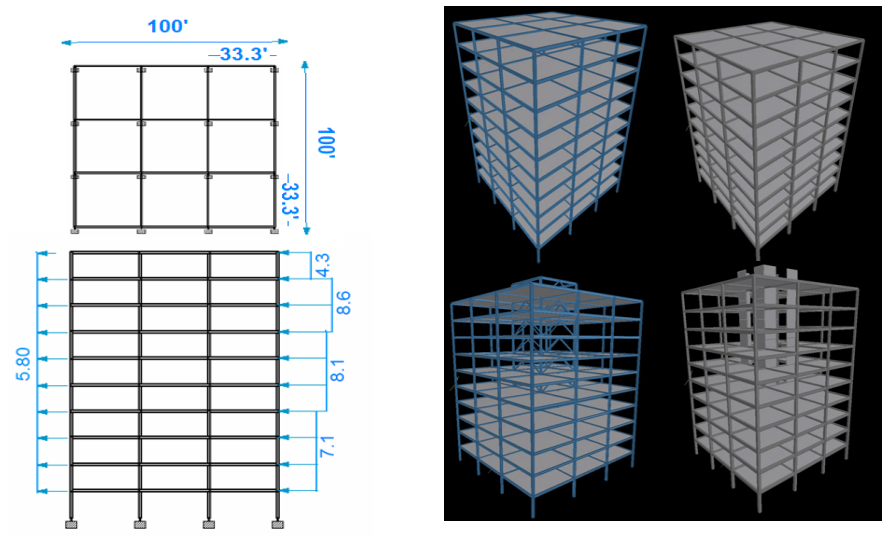

Figure 2: Wind loads in kips and perspective of 10-story building, (130') height with different structural systems. 
Table 1: $\quad$ Beams schedule for 10-story building for different structural systems.

\begin{tabular}{||c|c|c|c|c|c|c|c||}
\hline \hline \multicolumn{2}{||c|}{ Steel Moment Frame } & \multicolumn{2}{|c|}{ Steel Braced Frame } & \multicolumn{2}{l|}{ Conc. Moment Frame. } & \multicolumn{2}{c|}{ Concrete Shear wall } \\
\hline Beam Size & Floor No. & Beam Size & Floor No. & Beam Size & Floor No. & Beam Size & Floor No. \\
\hline W $18 \times 50$ & 9,10 & W $18 \times 50$ & 9,10 & $17.5^{\prime \prime} \times 12^{\prime \prime}$ & 9,10 & $18.0^{\prime \prime} \times 12^{\prime \prime}$ & 9,10 \\
\hline W $18 \times 60$ & 7,8 & W $18 \times 50$ & 7,8 & $19.0^{\prime \prime} \times 12^{\prime \prime}$ & 7,8 & $18.5^{\prime \prime} \times 12^{\prime \prime}$ & 7,8 \\
\hline W $18 \times 76$ & 5,6 & W $18 \times 55$ & 5,6 & $21.5^{\prime \prime} \times 12^{\prime \prime}$ & 5,6 & $21.0^{\prime \prime} \times 9.5^{\prime \prime}$ & 5,6 \\
\hline W $18 \times 76$ & 3,4 & W $18 \times 60$ & 3,4 & $22.0^{\prime \prime} \times 12^{\prime \prime}$ & 3,4 & $21.5^{\prime \prime} \times 9.5^{\prime \prime}$ & 3,4 \\
\hline W $18 \times 86$ & 1,2 & W $18 \times 65$ & 1,2 & $24.0^{\prime \prime} \times 12^{\prime \prime}$ & 1,2 & $24.0^{\prime \prime} \times 9.5^{\prime \prime}$ & 1,2 \\
\hline
\end{tabular}

Table 2: Columns schedule for 10-story building for different structural systems.

\begin{tabular}{|c|c|c|c|c|c|c|c|}
\hline \multicolumn{2}{|c|}{ Steel Moment Frame } & \multicolumn{2}{|c|}{ Steel Braced Frame } & \multicolumn{2}{|c|}{ Conc. Moment frame } & \multicolumn{2}{|c|}{ Concrete Shear wall } \\
\hline Column Size & Floor No. & Column Size & Floor No. & Column Size & Floor No. & Column Size & Floor No. \\
\hline W 14× 159 & 9,10 & W $14 \times 145$ & 9,10 & $17.5^{\prime \prime} \times 17.5^{\prime \prime}$ & 9,10 & $17.5^{\prime \prime} \times 17.5^{\prime \prime}$ & 9,10 \\
\hline W $14 \times 176$ & 7,8 & W $14 \times 159$ & 7,8 & $19.0^{\prime \prime} \times 17.5^{\prime \prime}$ & 7,8 & $18.0^{\prime \prime} \times 18.0^{\prime \prime}$ & 7,8 \\
\hline W $14 \times 211$ & 5,6 & W $14 \times 176$ & 5,6 & $20.5^{\prime \prime} \times 19.0^{\prime \prime}$ & 5,6 & $19.0 " \times 19.0^{\prime \prime}$ & 5,6 \\
\hline W $14 \times 283$ & 3,4 & W $14 \times 193$ & 3,4 & $20.5^{\prime \prime} \times 20.5^{\prime \prime}$ & 3,4 & $19.0^{\prime \prime} \times 20.5^{\prime \prime}$ & 3,4 \\
\hline W $14 \times 342$ & 1,2 & W $14 \times 233$ & 1,2 & $24.0^{\prime \prime} \times 24.0^{\prime \prime}$ & 1,2 & $21.0^{\prime \prime} \times 20.5^{\prime \prime}$ & 1,2 \\
\hline
\end{tabular}

\section{$5 \quad 20$-story building}
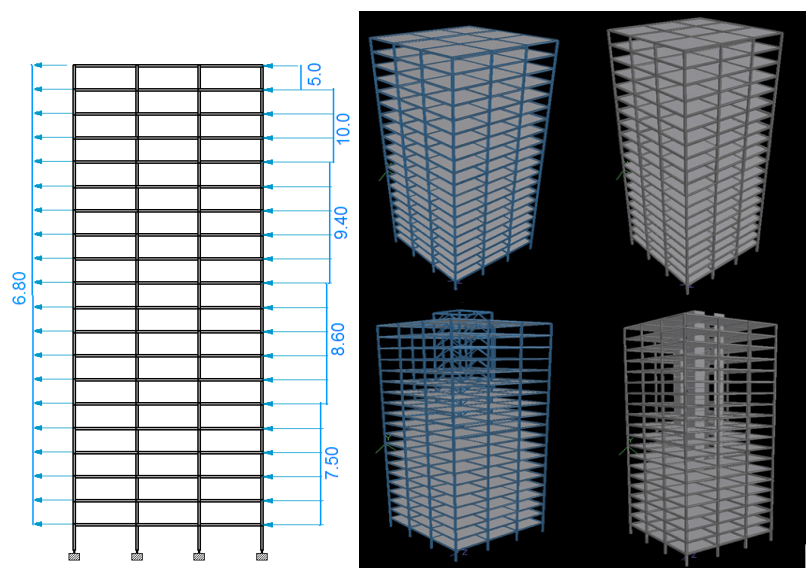

Figure 3: Wind load in kips and perspective of 20-story building, (260') height with different structural systems. 
Table 3: Beams schedule for 20-story building for different structural systems.

\begin{tabular}{|c|c|c|c|c|c|c|c|}
\hline \multicolumn{2}{|c|}{ Steel Moment Frame } & \multicolumn{2}{|c|}{ Steel Braced Frame } & \multicolumn{2}{|c|}{ Conc. Moment Frame } & \multicolumn{2}{|c|}{ Concrete Shear wall } \\
\hline Beam Size & Floor No. & Beam Size & Floor No. & Beam Size & Floor No. & Beam Size & Floor No. \\
\hline $\mathrm{W} 18 \times 55$ & 19,20 & W $18 \times 60$ & 19,20 & $18.0^{\prime \prime} \times 12.0^{\prime \prime}$ & 19,20 & $20.4 "$ x $12.0^{\prime \prime}$ & $17-20$ \\
\hline W $18 \times 71$ & 17,18 & W $18 \times 65$ & $16-18$ & $20.5^{\prime \prime} \times 12.0^{\prime \prime}$ & 17,18 & $21.0 " \mathrm{x} 13.2^{\prime \prime}$ & $14-16$ \\
\hline W $18 \times 86$ & 15,16 & W $18 \times 71$ & 14,15 & $21.6 " \times 13.2 "$ & 15,16 & $22.2^{\prime \prime} \times 13.2 "$ & 12,13 \\
\hline W $18 \times 97$ & 13,14 & W $18 \times 76$ & $1-13$ & $24.0 " \mathrm{x} 13.2 "$ & 13,14 & $22.8^{\prime \prime} \times 13.2^{\prime \prime}$ & 10,11 \\
\hline W $18 \times 106$ & 11,12 & ------------ & ------ & $24.0 " \mathrm{x} 14.4 "$ & 11,12 & $24.0 " \mathrm{\prime \prime} 13.2 "$ & $1-9$ \\
\hline W $18 \times 119$ & 9,10 & ------------ & ------ & $25.2 " \mathrm{x} 14.4 "$ & 9,10 & ---------- & ----- \\
\hline W $18 \times 130$ & $5,6,7,8$ & --------- & ------ & $27.0 " \mathrm{x} 14.4 "$ & $6,7,8$ & -------- & ב---- \\
\hline W $18 \times 143$ & $1,2,3,4$ & ----------- & -------- & $28.2 " \times 14.4 "$ & $1-5$ & ----------- & ----- \\
\hline
\end{tabular}

Table 4: Columns schedule for 20-story building for different structural systems.

\begin{tabular}{|c|c|c|c|c|c|c|c|}
\hline \multicolumn{2}{|c|}{ Steel Moment Frame } & \multicolumn{2}{|c|}{ Steel Braced Frame } & \multicolumn{2}{|c|}{ Conc. Moment Frame. } & \multicolumn{2}{|c|}{ Concrete Shear wall } \\
\hline Column Size & Floor No. & Column Size & Floor No. & Column Size & Floor No. & Column Size & Floor No. \\
\hline W14 x 159 & $18,19,20$ & W14 x 159 & $18,19,20$ & $18.0^{\prime \prime} \times 18.0^{\prime \prime}$ & $17-20$ & $18.0^{\prime \prime} \times 12.8^{\prime \prime}$ & $17-20$ \\
\hline $\mathrm{W} 14 \times 211$ & 16,17 & $\mathrm{~W} 14 \times 176$ & 16,17 & $19.0^{\prime \prime} \times 19.0^{\prime \prime}$ & 15,16 & $20.4 " \mathrm{~N} 12.0^{\prime \prime}$ & 15,16 \\
\hline W14 x 283 & 14,15 & W14 x 233 & 14,15 & $21.5^{\prime \prime} \times 20.5^{\prime \prime}$ & 13,14 & $20.4 "$ x 20.4" & 13,14 \\
\hline $\mathrm{W} 14 \times 342$ & 12,13 & W14 x 257 & 12,13 & $22.8^{\prime \prime} \times 22.8^{\prime \prime}$ & 11,12 & $22.2^{\prime \prime} \times 22.2^{\prime \prime}$ & 11,12 \\
\hline W14 x 426 & 10,11 & W14 x 311 & 10,11 & $25.2^{\prime \prime} \times 24.0^{\prime \prime}$ & 9,10 & $22.8^{\prime \prime} \times 22.8^{\prime \prime}$ & 9,10 \\
\hline $\mathrm{W} 14 \times 500$ & 8,9 & W14 × 342 & 8,9 & $25.2^{\prime \prime} \times 26.4 "$ & 7,8 & $18.0^{\prime \prime} \times 18.0^{\prime \prime}$ & 7,8 \\
\hline $\mathrm{W} 14 \times 550$ & $5-7$ & W14 x 370 & $5,6,7$ & $27.6^{\prime \prime} \times 27.6 "$ & 5,6 & $19.0^{\prime \prime} \times 19.0^{\prime \prime}$ & 5,6 \\
\hline W14 x 605 & 3,4 & W14 x 426 & 3,4 & $28.8^{\prime \prime} \times 28.8^{\prime \prime}$ & 3,4 & $19.0^{\prime \prime} \times 20.5^{\prime \prime}$ & 3,4 \\
\hline W $14 \times 730$ & 1,2 & W14 x 605 & 1,2 & $32.4 " \mathrm{x} \mathrm{32.4"}$ & 1,2 & $21.0^{\prime \prime} \times 20.5^{\prime \prime}$ & 1,2 \\
\hline
\end{tabular}

\section{$6 \quad 30$-story building}
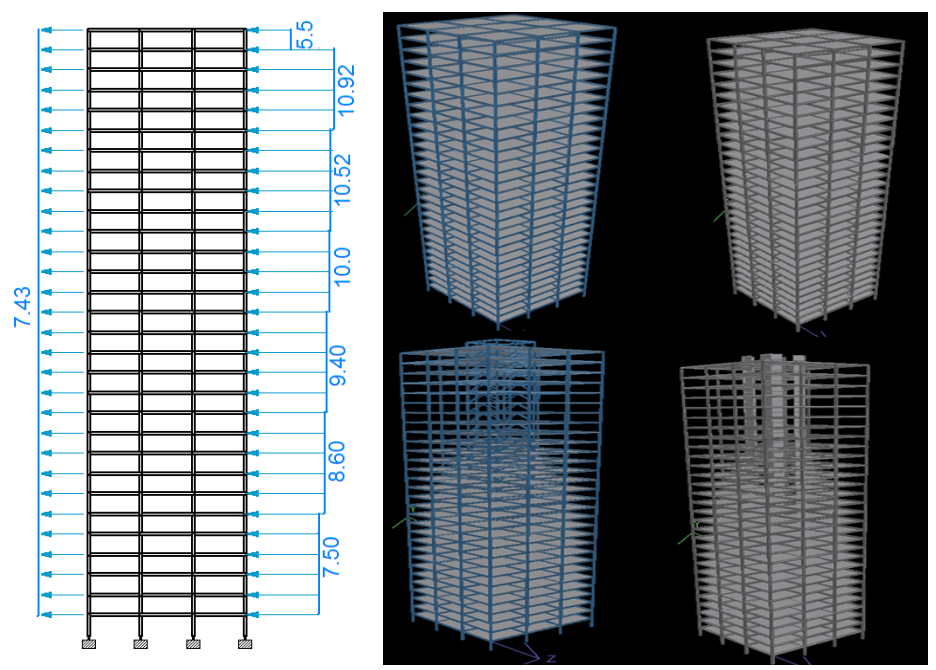

Figure 4: Wind loads in kips and perspective of 30-story building, (390') height with different structural systems. 
134 Computer Aided Optimum Design in Engineering X

Table 5: Beams schedule for 30-story building for different structural systems.

\begin{tabular}{|c|c|c|c|c|c|c|c|}
\hline \multicolumn{2}{|c|}{ Steel Moment Frame } & \multicolumn{2}{|c|}{ Steel Braced Frame } & \multicolumn{2}{|c|}{ Conc. Moment frame } & \multicolumn{2}{|c|}{ Conc. Shear wall } \\
\hline Beam Size & Floor No. & Beam Size & Floor No. & Beam Size & Floor No. & Beam Size & Floor No. \\
\hline W18 x 55 & 29,30 & W18 x 71 & 29,30 & $18.6^{\prime \prime} \times 12.0^{\prime \prime}$ & 29,30 & $19.8 " \mathrm{x} 14.4 "$ & 29,30 \\
\hline W18 x 71 & 27,28 & W18 x76 & 27,28 & $21.0^{\prime \prime} \times 12.0 "$ & 27,28 & $21 "$ x 14.4" & 27,28 \\
\hline W18 x 86 & 25,26 & W18 x 86 & $24,25,26$ & $21.6 " \mathrm{x} 14.4 "$ & 25,26 & $21 " \mathrm{x} 15.6 "$ & $24-26$ \\
\hline $\mathrm{W} 18 \times 106$ & 23,24 & W18 x 97 & 22,23 & $24.0 " \mathrm{x}$ 14.4" & 23,24 & $23.4 " x \quad 15.6 "$ & 22,23 \\
\hline W18 $\times 119$ & 21,22 & W18 $\times 106$ & $19,20,21$ & $25.2^{\prime \prime} \times 14.4^{\prime \prime}$ & 21,22 & $27 " \times 15.6 "$ & $19-, 21$ \\
\hline W18 $\times 130$ & 19,20 & W18x 119 & 17,18 & $27.0^{\prime \prime} \times 14.4 "$ & 19,20 & $28.8 " \mathrm{x} 16.8 "$ & $13-18$ \\
\hline W18 x 143 & $16,17,18$ & W18x 130 & 15,16 & $27.0 " \mathrm{n} 15.6 \mathrm{"}$ & 17,18 & $29.4 " \mathrm{x} 16.8 "$ & 11,12 \\
\hline W18 $\times 158$ & $13,14,15$ & W18x 119 & $1 \mathrm{TO} 14$ & $28.4 " \mathrm{~N} 15 . \mathrm{C}^{\prime \prime}$ & 15,16 & $30 " \times 16.8 "$ & $1 \mathrm{TO} 10$ \\
\hline W18 $\times 175$ & $9 \mathrm{TO} 12$ & ---------- & $\begin{array}{ll}-\cdots--- \\
-\cdots\end{array}$ & $29.0^{\prime \prime}$ x $16.8^{\prime \prime}$ & $10-14$ & 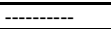 & |--- \\
\hline W18 x 192 & $6-8,1-3$ & 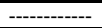 & 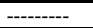 & $30.6^{\prime \prime} \times 18.0^{\prime \prime}$ & $1 \mathrm{~T} 09$ & |-----------י & ----- \\
\hline W18 $\times 211$ & 4,5 & ------------ & 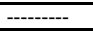 & $28.2 " \mathrm{x} 14.4 "$ & $1-5$ & |----------- & ----- \\
\hline
\end{tabular}

Table 6: Columns schedule for 30-story building for different structural systems.

\begin{tabular}{|c|c|c|c|c|c|c|c|}
\hline \multicolumn{2}{|c|}{ Steel Moment Frame } & \multicolumn{2}{|c|}{ Steel Braced Frame } & \multicolumn{2}{|c|}{ Conc. Moment frame. } & \multicolumn{2}{|c|}{ Conc. Shear wall } \\
\hline Column Size & Floor No. & Column Size & Floor No. & Column Size & Floor No. & Column Size & Floor No. \\
\hline W14 x 176 & $27-30$ & W14 x 176 & $27-30$ & $18.4^{\prime \prime}$ x 18.4" & $27-30$ & $18.6 " \mathrm{~m} 18.6^{\prime \prime}$ & $27-30$ \\
\hline W14 x 257 & 25,26 & W14 x 193 & 25,26 & $19.8^{\prime \prime}$ x 19.8" & 25,26 & $19.6 " \mathrm{~m} 19.6 "$ & 25,26 \\
\hline W14 x 342 & 23,24 & $\mathrm{~W} 14 \times 233$ & 23,24 & $21.7 " x$ 21.7" & 23,24 & $22.0^{\prime \prime} \times 22.0^{\prime \prime}$ & 23,24 \\
\hline W14 x 426 & 21,22 & W14 x 283 & 21,22 & $23.5^{\prime \prime} \times 23.5^{\prime \prime}$ & 21,22 & $23.4^{\prime \prime} \times 23.4 "$ & 21,22 \\
\hline W14 x 455 & 19,20 & $\mathrm{~W} 14 \times 342$ & 19,20 & $25.2^{\prime \prime} \times 25.2^{\prime \prime}$ & 19,20 & $24.6^{\prime \prime} \times 24.6 "$ & 19,20 \\
\hline W14 x 550 & $16,17,18$ & W14 x 370 & 17,18 & $26.6^{\prime \prime} \times 26.6^{\prime \prime}$ & 17,18 & $26.4 "$ x $26.4 "$ & 17,18 \\
\hline W14 x 605 & 14,15 & W14 x 398 & 15,16 & $28.2^{\prime \prime} \times 28.2^{\prime \prime}$ & 15,16 & $27.6^{\prime \prime} \times 27.6^{\prime \prime}$ & 15,16 \\
\hline W14 x 665 & 12,13 & W14 x 426 & 13,14 & $29.4^{\prime \prime} \times 29.4 "$ & 13,14 & $28.2^{\prime \prime}$ x 28.2" & 13,14 \\
\hline W14 x 730 & 10,11 & $\mathrm{~W} 14 \times 500$ & 11,12 & $30.6^{\prime \prime} \times 30.6^{\prime \prime}$ & 11,12 & $29.4 " x 29.4 "$ & 11,12 \\
\hline W14 x 825 & 8,9 & $\mathrm{~W} 14 \times 550$ & 9,10 & $32.4^{\prime \prime}$ x 32.4" & 9,10 & $30.0^{\prime \prime} \times 30.0^{\prime \prime}$ & $7-10$ \\
\hline W14 x905 & 6,7 & W14 x605 & 7,8 & $33.0^{\prime \prime} \times 33.0^{\prime \prime}$ & 7,8, & $30.6^{\prime \prime} \times 30.6^{\prime \prime}$ & 5,6 \\
\hline W14 x1025 & $3,4,5$ & W14 x665 & 5,6 & $33.6^{\prime \prime}$ x 33.6" & 5,6 & $31.2^{\prime \prime}$ x 31.2" & 3,4 \\
\hline W14 $\times 1105$ & 1,2 & W14 x 730 & 3,4 & $34.8^{\prime \prime} \times 34.8^{\prime \prime}$ & 3,4 & $33.6^{\prime \prime} \times 33.6^{\prime \prime}$ & 1,2 \\
\hline -------- & ------ & W14 x 825 & 1,2 & $39.3^{\prime \prime}$ x 39.3" & 1,2 & ---------- & $-\cdots$ \\
\hline
\end{tabular}

\section{40-story building}

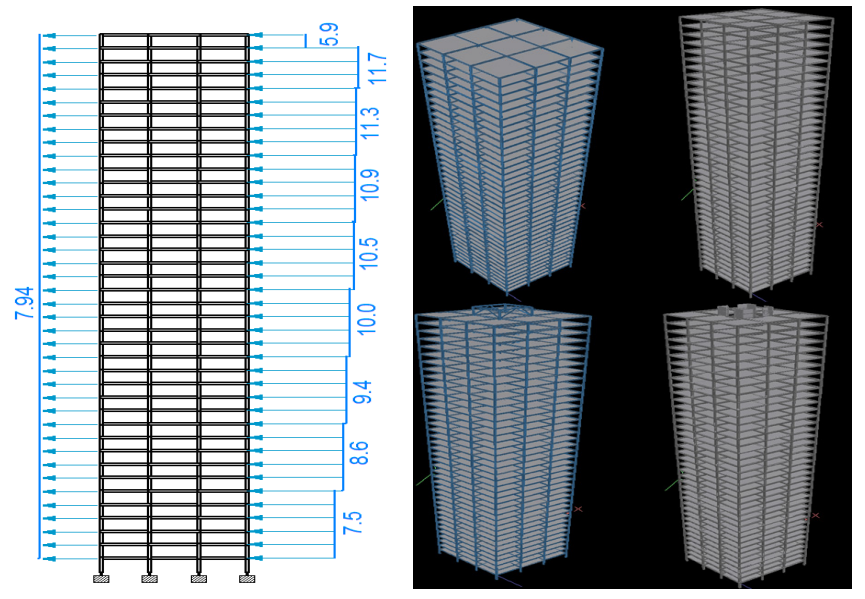

Figure 5: Wind loads in kips and perspective of 40-story building, (520') height with different structural systems. 
Table 7: Beams schedule for 40-story building for different structural systems.

\begin{tabular}{|c|c|c|c|c|c|c|c|}
\hline \multicolumn{2}{|c|}{ Steel Moment Frame } & \multicolumn{2}{|c|}{ Steel Braced Frame } & \multicolumn{2}{|c|}{ Conc. Moment frame } & \multicolumn{2}{|c|}{ Concrete Shear wall } \\
\hline Beam Size & Floor No. & Beam Size & Floor No. & Beam Size & Floor No. & Beam Size & Floor No. \\
\hline W18 x 55 & 39,40 & $\mathrm{~W} 18 \times 71$ & 39,40 & $18.6 "$ x 12" & 39,40 & $19.2 " \mathrm{e} \mathrm{14.4"}$ & $38-40$ \\
\hline W18 x76 & 37,38 & W18 X76 & 37,38 & $21 " \times 12 "$ & 37,38 & $20.4 " \mathrm{n} 14.4 "$ & 36,37 \\
\hline $\mathrm{W} 18 \times 97$ & 35,36 & $\mathrm{~W} 18 \times 86$ & 35,36 & $21.6 " \mathrm{"} \times 14.4 "$ & 35,36 & $22.2^{\prime \prime} \times 15.6^{\prime \prime}$ & 34.35 \\
\hline $\mathrm{W} 18 \times 106$ & 33,34 & W18 x 97 & 33,34 & $24 "$ x 14.4" & 32,33 & $23.4 " \mathrm{x} 15.6 "$ & 32,33 \\
\hline W18x 119 & 31,32 & W18 $\times 119$ & $30,31,32$ & $25.8 " \times 14.4 "$ & 30,31 & $24 " \times 16.8 "$ & 30,31 \\
\hline W18x 143 & 29,30 & W18x 130 & 28,29 & $28 " \mathrm{x} 14.4 "$ & 28,29 & $25.2^{\prime \prime} \times 16.8^{\prime \prime}$ & $27-28$ \\
\hline W18x 158 & $25-28$ & W18x 43 & 7 TO 10 & $28 " \times 15.6 "$ & 26,27 & $30^{\prime \prime} \times 16.8 "$ & 25,26 \\
\hline W18x 175 & 23,24 & W18x 158 & $23-27$ & $28.8^{\prime \prime} \times 15.6 "$ & 24,25 & $32.4^{\prime \prime} \times 16.8^{\prime \prime}$ & $21-24$ \\
\hline W18x 192 & $20-, 22$ & W18x 175 & 18 TO 22 & $29.4 " \mathrm{"} \mathrm{x} \mathrm{16.8"}$ & 22,23 & $33.6 " \mathrm{"} \mathrm{x} 18 \mathrm{"}$ & $15-20$ \\
\hline W18x 211 & $16-19$ & W18x 192 & $15-17$ & $31.2^{\mathrm{N}} \times 16.8^{\mathrm{\prime}}$ & $18-20$ & $32.4 " \mathrm{"} \times 16.8^{\prime \prime}$ & $11-14$ \\
\hline W18x 234 & $12-15$ & W18x 158 & $11-14$ & $32.4^{\prime \prime}$ x $18 "$ & $11-17$ & $33.6 "$ x $18 "$ & $6-10$ \\
\hline W18x 258 & $1 \mathrm{TO} 11$ & W18x 130 & $1 \mathrm{TO} 6$ & $35.4 " \times 18^{\prime \prime}$ & $1-10$ & $32.4 " \mathrm{n} 16.8^{\prime \prime}$ & $1 \mathrm{TO} 5$ \\
\hline
\end{tabular}

Table 8: Columns schedule for 40-story building for different structural systems.

\begin{tabular}{|c|c|c|c|c|c|c|c|}
\hline \multicolumn{2}{|c|}{ Steel Moment Frame } & \multicolumn{2}{|c|}{ Steel Braced Frame } & \multicolumn{2}{|c|}{ Conc. Moment Frame } & \multicolumn{2}{|c|}{ Concrete Shear wall } \\
\hline Column Size & Floor No. & Column Size & Floor No. & Column Size & Floor No. & Column Size & Floor No. \\
\hline W14 x 176 & 37 TO 40 & W14 x 193 & 37 TO 40 & $18.4^{\prime \prime} \times 18.4 "$ & 37 TO 40 & $19.2^{\prime \prime} \times 19.2^{\prime \prime}$ & $37-40$ \\
\hline W14 x 257 & 35,36 & W14 x 211 & 35,36 & $20 " \times 20 "$ & 35,36 & $20 " \times 20 "$ & 35,36 \\
\hline W14 x 342 & 33,34 & W14 $\times 283$ & 33,34 & $22 "$ x 22" & 33,34 & $21 " \times 21 "$ & 33,34 \\
\hline W14 x 426 & 31,32 & $\mathrm{~W} 14 \times 342$ & 31,32 & $24 "$ x $24 "$ & 31,32 & $22.5^{\prime \prime} \times 22.5^{\prime \prime}$ & 31,32 \\
\hline $\mathrm{W} 14 \times 500$ & 29,30 & W14 x 370 & 29,30 & $25.6^{\prime \prime} \times 25.6 "$ & 29,30 & $24 "$ " x24" & 29,30 \\
\hline $\mathrm{W} 14 \times 550$ & 27,28 & W14 x 426 & 27,28 & $27 " \times 27 "$ & 27,28 & $25.2^{\prime \prime}$ x 25.2" & 27,28 \\
\hline W14 x 605 & 25,26 & W14 x 500 & 23 TO 26 & $28.8^{\prime \prime}$ x 28.8" & 25,26 & $27.4 "$ x 27.4" & 25,26 \\
\hline $\mathrm{W} 14 \times 730$ & $21 \mathrm{TO} 24$ & $\mathrm{~W} 14 \times 550$ & 21,22 & $30 "$ x 30" & 23,24 & $29.4 "$ x 29.4" & 23,24 \\
\hline W14 x 865 & 19,20 & W14 x 605 & 17 TO 20 & $31.2^{\prime \prime} \times 31.2^{\prime \prime}$ & 21,22 & $30^{\prime \prime} \times 30^{\prime \prime}$ & 21,22 \\
\hline W14 X 905 & 17,18 & W14 x 665 & 15,16 & $32.4 " \mathrm{n} 32.4 "$ & 19,20 & $31.2^{\prime \prime} \times 31.2^{\prime \prime}$ & 19,20 \\
\hline W14 x 985 & 15,16 & W14 x730 & 13,14 & $33.6^{\prime \prime} \times 33.6 "$ & 17,18 & $32.4^{\prime \prime} \times 32.4 "$ & 17,18 \\
\hline W14 x 1065 & 13,14 & W14 x825 & 11,12 & $34.2^{\prime \prime} \times 34.2^{\prime \prime}$ & 15,16 & $33 " \times 33 "$ & 15,16 \\
\hline W14 × 1145 & 11,12 & W14 x 1225 & 9,10 & $35.4 "$ x 35.4" & 13,14 & $33.6 "$ x $33.6 "$ & 11to14 \\
\hline W14 x 1265 & 9,10 & W14 X 1305 & 7,8 & $36.4 "$ x 36.4" & $10,11,12$ & $34.2 \mathrm{n}$ x 34.2" & 9,10 \\
\hline W14 x 1305 & 7,8 & W14 x 1385 & 5,6 & $37.4 "$ x 37.4" & $7,8,9$ & $34.6 "$ x $34.6 "$ & 7,8 \\
\hline W14 x 1385 & 5,6 & W14 x 1465 & 3,4 & $38.7^{\prime \prime} \times 38.7^{\prime \prime}$ & 5,6 & $35.4 " \mathrm{n} 35.4 \mathrm{"}$ & 5,6 \\
\hline W14 x 1465 & 3,4 & W14 x 1545 & 1,2 & $39.6 " \mathrm{x} 39.6^{\prime \prime}$ & 3,4 & $36 " \times 36 "$ & 3,4 \\
\hline W14 X 1545 & 1,2 & |----------- & -------- & $46 " \times 46 "$ & 1,2 & 38.4" x 38.4" & 1,2 \\
\hline
\end{tabular}




\section{Lateral drift comparison}
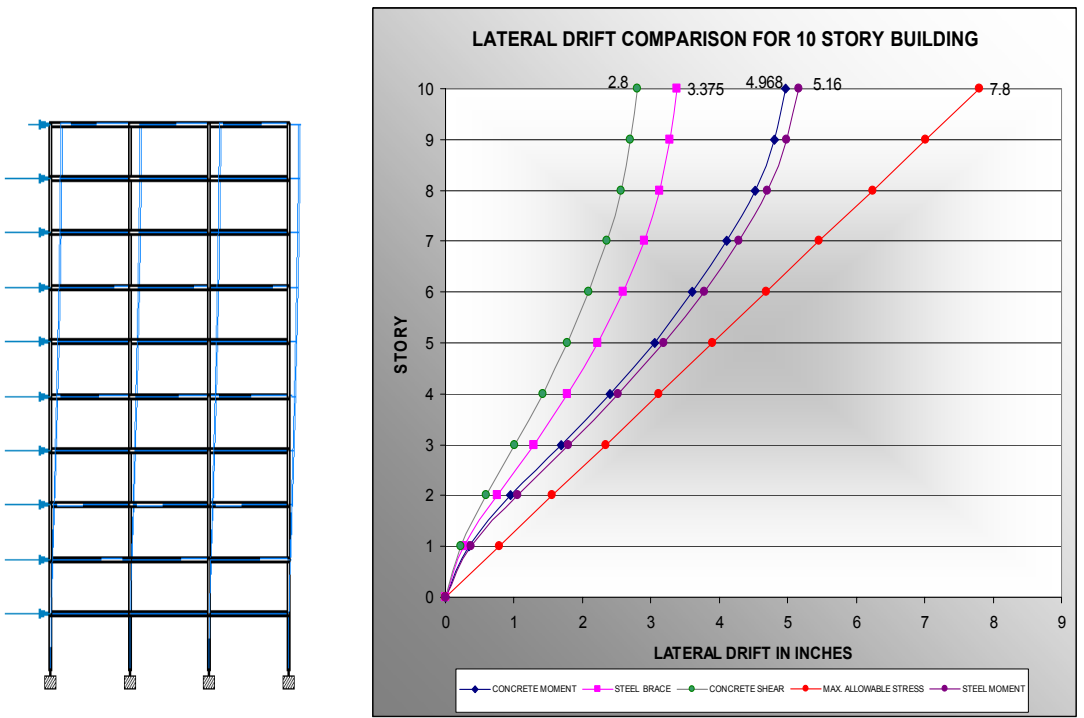

Figure 6: Lateral drift comparison for 10-story building.
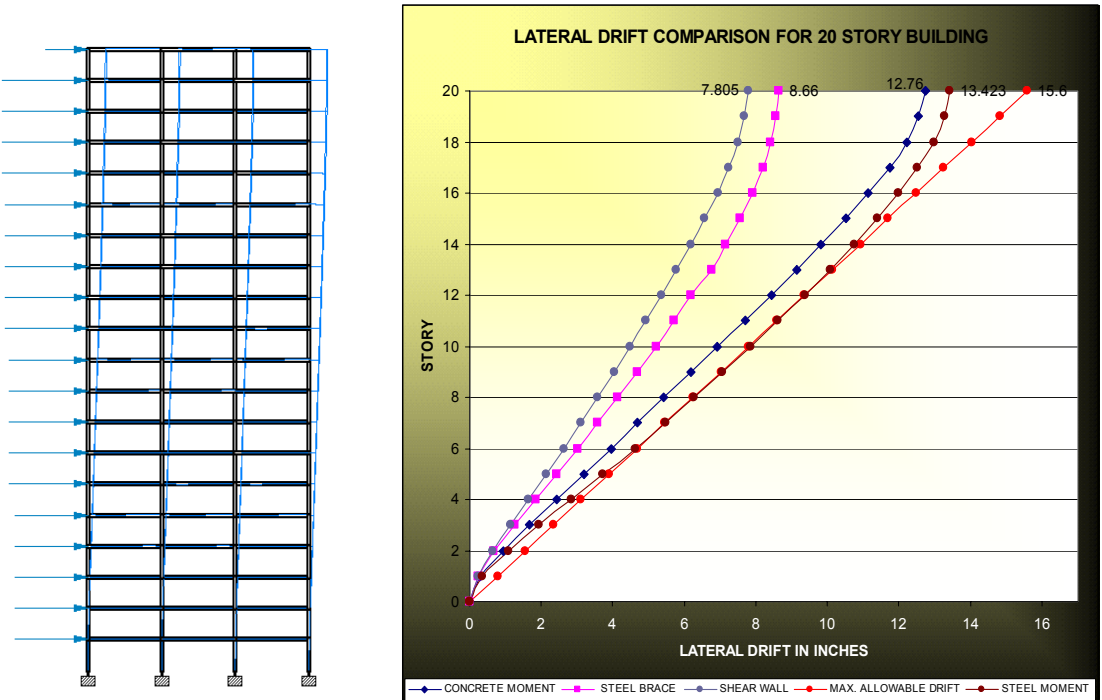

Figure 7: $\quad$ Lateral drift comparison for 20-story building. 

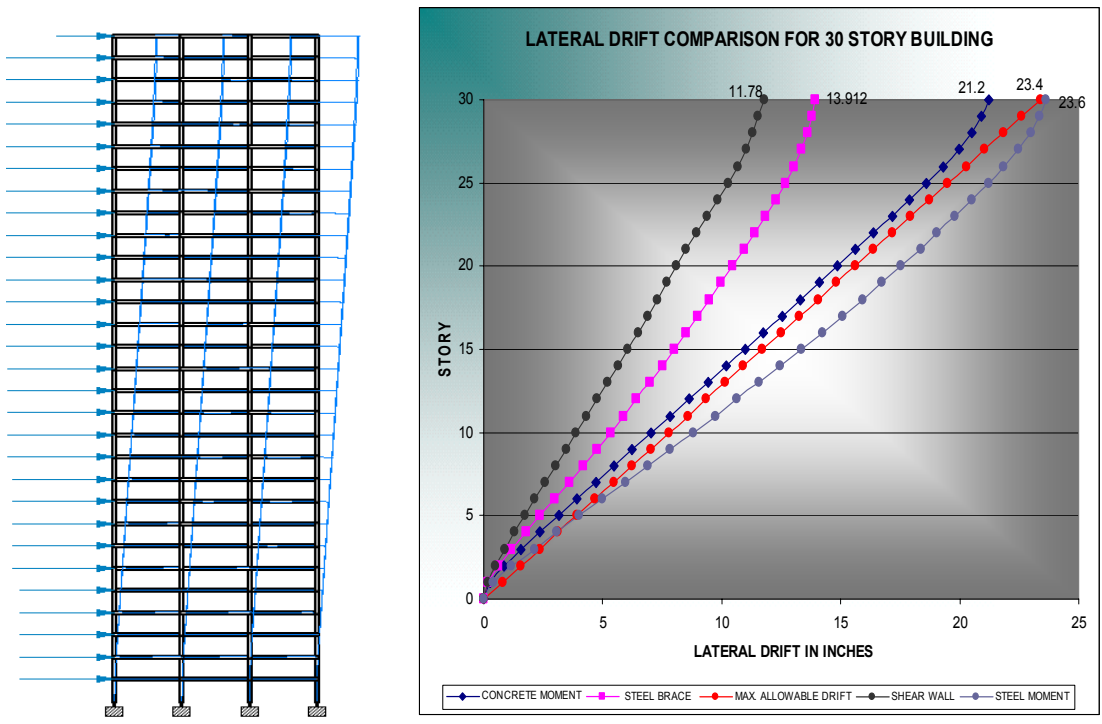

Figure 8: $\quad$ Lateral drift comparison for 30-story building.
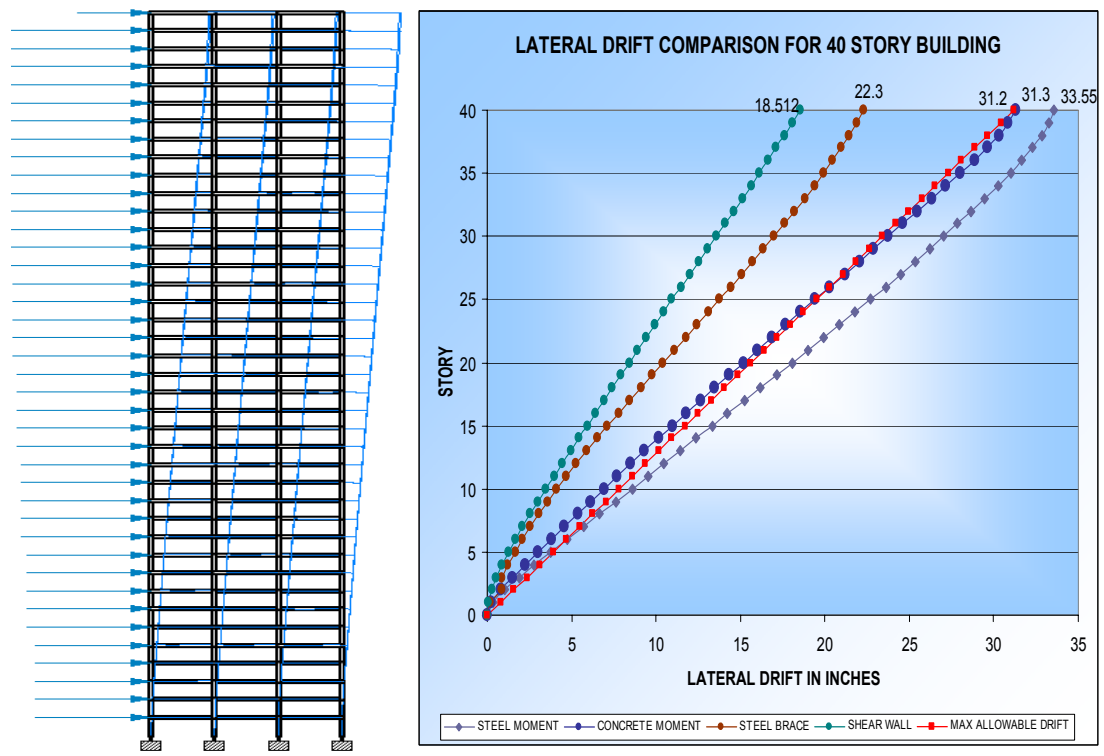

Figure 9: $\quad$ Lateral drift comparison for 40-story building. 


\section{Cost comparison}

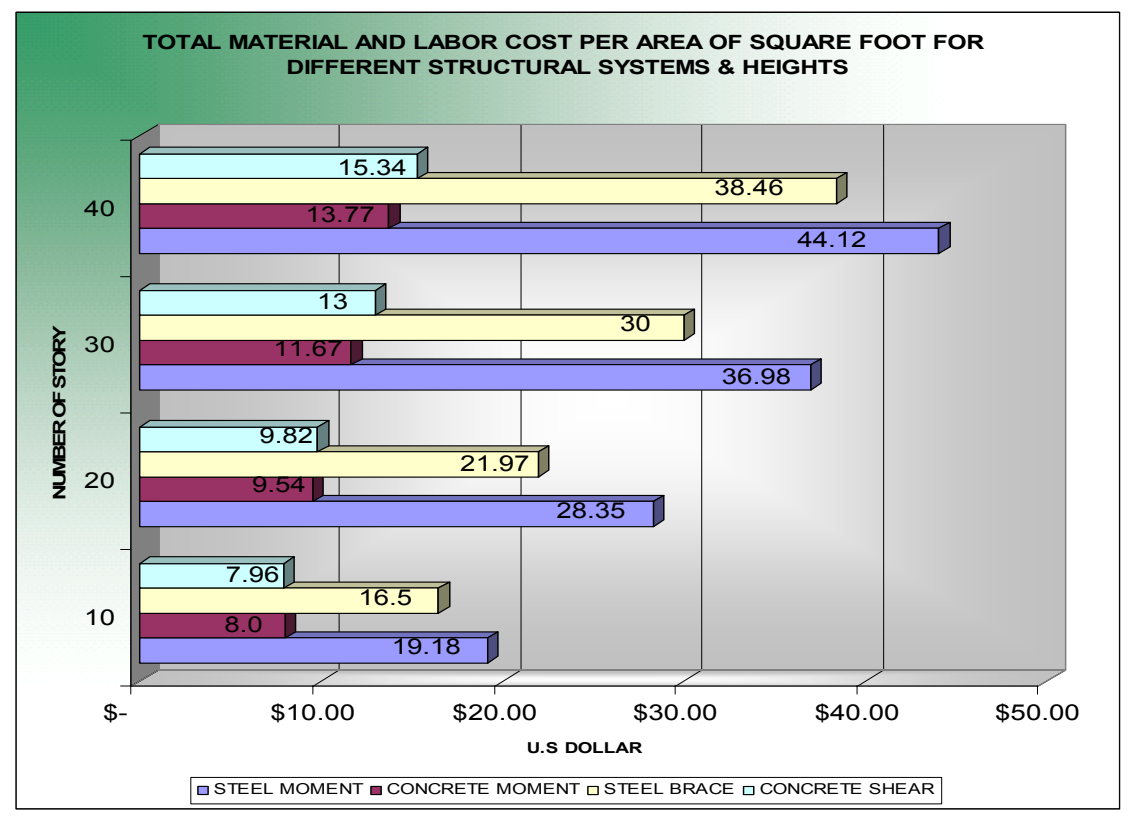

Figure 10: Cost comparison for structural systems.

\section{Results}

Comparing lateral drift and the cost for various structural systems at different heights shows the following results:

Figure 6 shows that the optimum structural system for a 10 -story building is concrete shear wall with lateral drift (2.8"). The concrete shear wall minimizes drift in comparison to the maximum allowable drift by $64 \%$, steel braced (3.4") reduces the drift by $57 \%$, concrete moment (4.9") reduces by $37 \%$, steel moment (5.6") reduces by $34 \%$ in comparison to the maximum allowable drift (7.8").

Figure 7 shows that the optimum structural system for a 20 -story building is concrete shear wall with lateral drift (7.8"). It minimizes lateral drift by $50 \%$, steel braced (8.7") reduces by $44 \%$, concrete moment (12.8") reduces by $18 \%$, steel moment (13.4") reduces by $14 \%$ in comparison to the maximum allowable drift (15.6").

Figure 8 shows that the optimum structural system for a 30 -story building is concrete shear wall with lateral drift (11.8"). It minimizes lateral drift in comparison to the maximum allowable drift by $50 \%$, steel braced (13.9") reduces the drift by $41 \%$, concrete moment (21.2") reduces by $9 \%$, steel moment $\left(23.6^{\prime \prime}\right)$ exceeds drift by $(-1 \%)$ in comparison to the maximum allowable drift $(23.4 ")$. 
Figure 9 shows that the optimum structural system for a 40 -story building is concrete shear wall with lateral drift (18.5") minimizes lateral drift in comparison to the maximum allowable drift by $41 \%$, steel brace (22.3") reduce $28 \%$, concrete moment (31.3") exceeds drift by $(-0.5 \%)$, steel moment (33.5") exceeds drift by (-8\%) in comparison to the maximum allowable drift (31.2").

Figure 10 shows that the optimum structural system for a 10 -story building to minimize the cost is shear wall $\left(7.96 \$ / \mathrm{ft}^{2}\right)$. It reduces the cost by $59 \%$ in comparison steel moment which has the highest cost, $\left(19.18 \$ / \mathrm{ft}^{2}\right)$.

The optimum structural system for 20-, 30-, and 40-story building is concrete moment $\left(9.54 \$ / \mathrm{ft}^{2}\right),\left(11.67 \$ / \mathrm{ft}^{2}\right)$, and $\left(13.77 \$ / \mathrm{ft}^{2}\right)$. It reduces the cost by $66 \%$, $68 \%$, and $69 \%$ as follows in comparison to the highest cost, steel moment $\left(28.35 \$ / \mathrm{ft}^{2}\right),\left(36.98 \$ / \mathrm{ft}^{2}\right)$, and $\left(44.12 \$ / \mathrm{ft}^{2}\right)$.

A steel braced frame reduces the cost by $14 \%, 23 \%, 19 \%$, and $13 \%$ for 10 , 20, 30, and 40 stories in comparison to steel moment frame.

For a 10-story building in Saudi Arabia, steel building costs twice more than concrete building.

For 20-, 30-, and 40-story building in Saudi Arabia, a steel building costs three times more than concrete building.

\section{Conclusions}

Using the proposed process is a vital method to select the optimum structural system to minimize lateral drift and cost. In Saudi Arabia shear wall is the optimum structural system. Concrete proves to be the optimum structural material to minimize lateral drift and reduce material and labour costs in comparison to steel. Adding shear walls in the concrete building is important to reduce lateral drift. This investigation shows that adding shear wall to concrete moment frame reduces lateral drift by $50 \%$ of the maximum allowable drift. Adding braces to steel moment frame minimizes the lateral drift, the cost, and the building mass. Drift governs the design when the height exceeds 30 stories for the steel moment frame and 40 stories for the concrete moment frame.

\section{References}

[1] G. G Schierle, Structures in Architecture, School of Architecture, University of Southern California, 2006.

[2] IBC 03 International Building Code, Internal Code Council, Fall Church, Virginia, 2003.

[3] AISC Manual of Steel Construction Allowable Stress Design, American Institute of Steel Construction, Chicago, ninth edition, 1991.

[4] James Ambrose and Dimitry Vergun, Design for Lateral Forces, 1987 by John Wiley \& Sons, Inc.

[5] CRSI Concrete Reinforcing Steel Institute, Guide to Structural System Selection, 1997. 
[6] Cheng-Yu Ho, Lateral Drift Cause by Wind Forces in High-Rise Steel Frame Master of Building Science Thesis, University of Southern California, August 1992.

[7] Cheng-Yu Ho and G. G Schierle, High-Rise Space Frame: Effect of Configuration on Lateral Drift, Preceding, $4^{\text {th }}$ International Conference on Space Structures, Surrey, Telford Publications.

[8] Eng. Riyadh ALhindi, Concrete cost in Saudi Arabia, Saudi Ready Mix Company.

[9] Eng. Ahmad Salah, Steel cost in Saudi Arabia, Zamil Steel Industries.

[10] Eng. Abduljalil Zaid, Construction Cost in Dammam, Saudi Arabia, Tamimi Trading and Contracting Co. Ltd. (TIMCO). 